\title{
A Hybrid Scheme Motion Controller by Sliding Mode and Two-Degree-of-Freedom Controls to Minimize the Chattering
}

\author{
Chiu-Keng Lai \\ Department of Electrical Engineering, National Chin-Yi University of Technology, No. 57, Section 2, Zhongshan Road, \\ Taiping District, Taichung 41170, Taiwan \\ Correspondence should be addressed to Chiu-Keng Lai; chiukl@ncut.edu.tw
}

Received 2 March 2014; Accepted 8 April 2014; Published 19 May 2014

Academic Editor: Her-Terng Yau

Copyright (C) 2014 Chiu-Keng Lai. This is an open access article distributed under the Creative Commons Attribution License, which permits unrestricted use, distribution, and reproduction in any medium, provided the original work is properly cited.

Sliding mode control (SMC) is rapped for the chattering due to high gain control. However, high gain control causes the system robust. For developing a system with robustness of SMC, a servo motor motion controller combining the two-degree-of-freedom (2DOF) system and SMC is proposed. The discussed motion type is point-to-point control with the constraint of trapezoid velocity profile. SMC is designed to guide the motor motion to follow a predefined trail, and the inner 2DOF system is used to compensate the deterioration due to the adoption of load observer. The proposed hybrid system is realized on a PC-based motion controller, and the validness is verified by simulation and experimental results.

\section{Introduction}

The control of motors used in high-performance servo drive requires that the prescribed torque accuracy, velocity, and position for all operating conditions should be achieved. Nevertheless, a precision model is difficult to construct because of the inherent nonlinearity of friction and dead zone, the parameter variations from temperature, and so on. Also, the time-varying loads often exist in the motor system, which cause those effects of increasing rise time, settling time, and percentage of overshoot in transient and steady state. To design a robust controller with those abilities to compensate the nonlinearity and uncertainties, minimizing the effects from external load is requisite for precision control.

Sliding mode control (SMC) is a very effective method to make a system robust and have fast responses [1]. It is thus widely practiced in the position and velocity control of DC and AC motor drives [2-8]. However, systems controlled by SMC will raise the phenomenon of chattering due to the finite switching frequency of relay controller, the high gain design, and the unmodeled system parameters $[9,10]$. Finite switching frequency is an inherent problem because the modern controllers are usually implemented with the microprocessor, PC or DSP, which runs program at a finite sampling frequency. As for the high gain design, it is certainly used to guarantee the existence of sliding phase, and its magnitude is usually devised according to the known upper bound of the external load and uncertainty of controlled system. In the case of the third reason of chattering, the unmodeled system may result from the reduced order approximation of system [9] or the occurrence of parasitic dynamics [11]. And a chatter input to the drive may excite the unmodeled poles and cause the system unstable or oscillated. Also, the disturbance and nonlinearities of motor controlled system, such as dead band and backlash, may also prevail in the steady-state mode. Take the precision control into consideration; those nonlinearities cause the system control law to be unremovable when the motor is in steady-state phase. The chatter control thus lasts for all the process time while the system is controlled by SMC. Therefore, a proper control law to overcome the external load and nonlinearities is essential during the period, including the sliding phase and steady-state phase. And one expects that the chatter control is slight enough to lower the chattering and avoid the system oscillating. Some recent developing strategies to reduce the chattering are introduced in $[6-8,10$, 12]. In [6], the authors deal with the torque ripple reduction by using a sliding mode control technique to the direct torque control of permanent magnet synchronous motor. In $[7,8]$, the authors develop the disturbance observer or load torque observer to reduce the chattering. The authors 
in [10] take the time constant of the actuator, the step size of the real time solver, and so forth into consideration to reduce the chattering for incremental sliding mode controller. While, in [12], gain scheduling is adopted to reduce the chattering effects, here, we adopt a hybrid structure to diminish the chattering. Hybrid structures used to the motor motion control can be found in [12-14], which combine those controllers such as PID controller and adaptive controller to get the desired performances.

It is known that the $2 \mathrm{DOF}$ system also has the robust ability and is sometimes used to regulate the system performance on transient and steady state. Because the chattering is mostly caused by a high gain control, we thus arrange an observer to estimate the load, and a $2 \mathrm{DOF}$ scheme is added as the inner loop to somewhat reduce the magnitude of external disturbance and parameters uncertainty. Regarding the proposed control structure, the control law can be reduced and the system's robustness is still valid.

The paper is organized in the following manner. The servo motor motion control is first shown in Section 2; next, Section 3 describes the strategy to estimate the perturbations; Section 4 points out the new structure with the 2DOF control being added in the inner loop; the simulation results which combine the SMC and 2DOF are revealed in Section 5; then, the experimental setup and results of permanent magnet synchronous motor drive position control are illustrated in Section 6; and finally, the conclusion is given in Section 7.

\section{The Servo Motor Motion Control with Sliding Mode System}

The rotor dynamics and the torque equation of motor plus driver are given as follows:

$$
\begin{gathered}
\frac{d \theta_{m}}{d t}=\omega_{m}, \\
\frac{d \omega_{m}}{d t}=-\frac{B_{m}}{J_{m}} \omega_{m}+\frac{T_{e}}{J_{m}}-\frac{T_{L}}{J_{m}}, \\
T_{e}=K_{t} v
\end{gathered}
$$

where $\omega_{m}$ is the rotor velocity, $\theta_{m}$ is the rotor angular displacement, $J_{m}$ is the moment of inertia, $B_{m}$ is the damping coefficient, $T_{e}$ is the generated torque, $K_{t}$ is the torque constant, $v$ is the control input to the motor driver, and $T_{L}$ is the external load. It is noted that the notations of the moment of inertia $J_{m}$ and the damping coefficient $B_{m}$ symbolize their normal values, $\bar{J}_{m}$ and $\bar{B}_{m}$, plus the uncertainties, $\Delta J_{m}$ and $\Delta B_{m}$; that is,

$$
\begin{gathered}
J_{m}=\bar{J}_{m}+\Delta J_{m}, \\
B_{m}=\bar{B}_{m}+\Delta B_{m} .
\end{gathered}
$$

The point-to-point incremental motion control is to move a resting object at time $t_{0}$ to a fixed desired position $\theta_{d}$ at time $t_{d}$ and then keep still until the next motion is initiated. In such process, the controlled system is not only subjected to the desired velocity and acceleration, but also affected by those conditions such as the uncertainties of parameter $\left(\Delta J_{m}\right.$ and $\left.\Delta B_{m}\right)$, the external applied load $\left(T_{L}\right)$, and the nonlinearities of the system (saturation, dead band, etc.). So the motion control is carried out under velocity control in obedience to a desired velocity mode. To fulfill the velocity and acceleration limitations, a trapezoid velocity profile is usually used or discussed $[2,3,13,15]$, as shown in Figure 1. In the acceleration and deceleration segment, the motor responds to a constant acceleration; in the run segment, on the other hand, it runs in the condition of constant velocity.

Because the system dynamics is subjected to unknown load perturbations, parameter variations, and system's nonlinearities, it is not easy to control the motor motion in accordance with the given trapezoid profile. A robust motion controller based on the SMC system is consequently adopted to process the velocity and acceleration constraint. To reach this goal, the multisegment sliding mode controller according to the trapezoid velocity profile is designed in advance with (i) acceleration segment (acceleration $=\alpha_{d 1}$ ), (ii) run segment (velocity $=$ $\omega_{d}$ ), and (iii) deceleration segment (deceleration $=-\alpha_{d 3}$ ). Therefore, the motor rotates in obedience to the trapezoid velocity profile in spite of the load perturbation and system's nonlinearities. The general control structure is shown in Figure 2(a).

With a designated rotor position $\theta_{d}$, which is supposed to be a constant within the control process for point-to-point control, one first defines the position error and its derivative as

$$
\begin{aligned}
& x_{1}=\theta_{m}-\theta_{d} \\
& x_{2}=\omega_{m} .
\end{aligned}
$$

Combining (3) with (1), one obtains

$$
\begin{aligned}
& \dot{x}_{1}=x_{2} \\
& \dot{x}_{2}=-\frac{B_{m}}{J_{m}} x_{2}+\frac{K_{t}}{J_{m}} v-\frac{T_{L}}{J_{m}} .
\end{aligned}
$$

Based on the error dynamical equations in (4), the multisegment sliding mode controller is proposed to drive the motor from initial position $\theta_{0}$ to the specified position $\theta_{d}$ according to the trapezoid velocity profile.

The multisegment SMC-based motion control system consists of two modes: the velocity control one and the position control one. The former is used to drive the rotor to the desired position while the latter is to hold the rotor at the desired position.

Based on SMC strategy, four sliding surfaces are assigned to guarantee the velocity and acceleration fitting the desired trapezoidal velocity profile [3].

\section{The Chattering Reduction with Perturbation Estimation}

As mentioned in the introduction and shown on most of the control problems solved by SMC, the chattering phenomenon must be reduced to improve the steady-state performance 


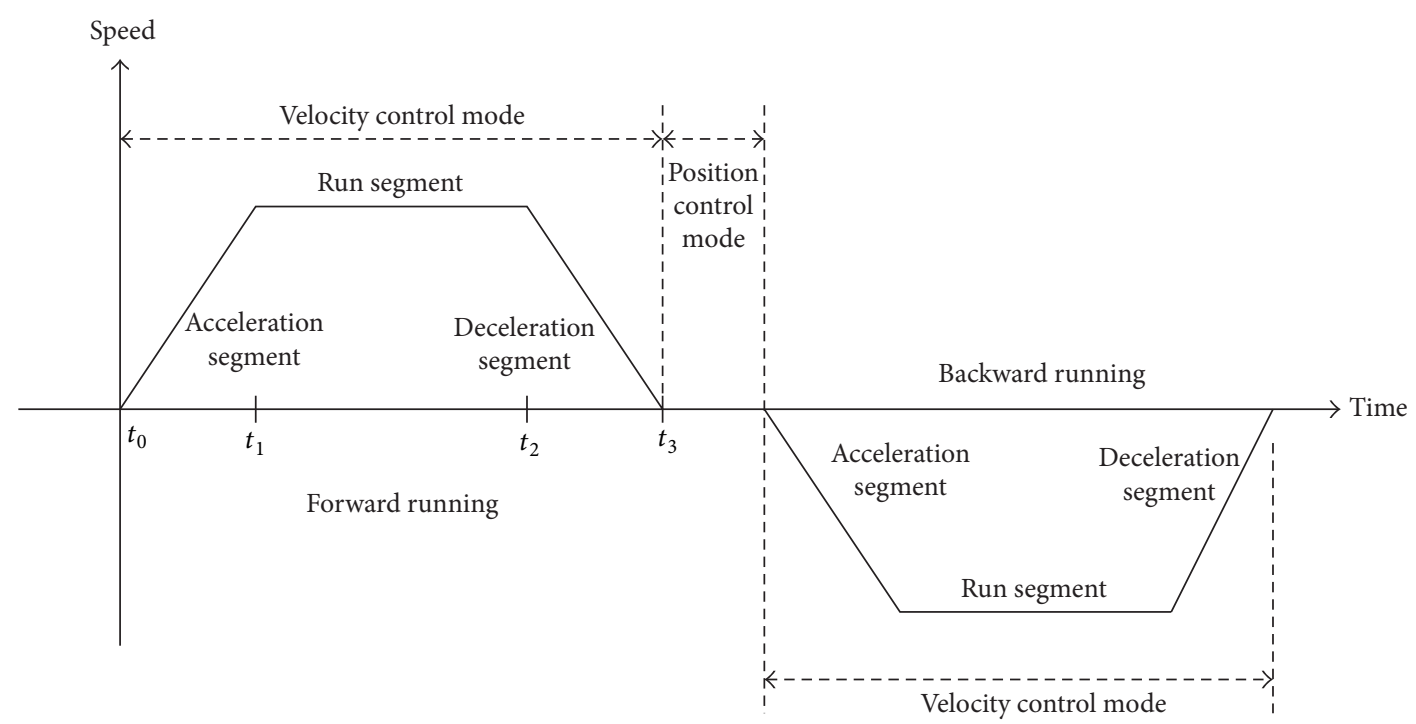

FIgURE 1: Trapezoid velocity profile for motion control.

and to prevent the unmodeled system from exciting. In the following, we will give the strategy to estimate the total perturbation and use the estimated results to reduce the chattering for our control requirement.

The description and design of multisegment sliding mode motion controller shown in Section 2 are summarized as follows.

(1) The reaching phase does not appear in the proposed multisegment sliding mode control method; that is, the system controlled by the proposed one resides in the sliding mode at the start and in the subsequent processes.

(2) For system in the acceleration/deceleration segment, the motor's dynamics can be simplified to the following form:

$$
\begin{aligned}
T_{e} & =K_{t} \nu \\
& =J_{m} \frac{d \omega_{m}}{d t}+B_{m} \omega_{m}+T_{L} \\
& =B_{m} \omega_{m}+J_{m} \alpha_{d}+T_{L}, \\
\frac{T_{L}}{K_{t}} & =v-\frac{B_{m}}{K_{t}} \omega_{m}-\frac{J_{m}}{K_{t}} \alpha_{d} .
\end{aligned}
$$

And for system in the run action, the motor's dynamics is simplified to be

$$
\begin{gathered}
T_{e}=K_{t} v=B_{m} \omega_{m}+T_{L}, \\
\frac{T_{L}}{K_{t}}=v-\frac{B_{m}}{K_{t}} \omega_{m} .
\end{gathered}
$$

Finally, once the system resides in the position control mode, the dynamics can be further simplified as

$$
\begin{gathered}
T_{e}=K_{t} v=T_{L}, \\
\frac{T_{L}}{K_{t}}=v .
\end{gathered}
$$

Thus, the load estimator is designed as

$$
\frac{d}{d t} v^{*}=-a v^{*}+a v
$$

where $v^{*}$ is the estimated equivalent control law, $v$ is the original chatter control law from SMC controller and it has a bigger magnitude, and $a$ is the estimator parameter to be designed. Due to the system in the sliding mode at the start, the estimated control law $v^{*}$ is initially equal to the equivalent control and all the proceeding time. The control law $v^{*}$ from observer output therefore has the least requirement for maintaining the system motion following the required trajectory. However, it is the same as most of the chattering reduction methodology of SMC, and the system robustness will be deteriorated by the parameter variation. To compensate the fault, $2 \mathrm{DOF}$ scheme is thus designed to compensate it.

\section{The Inner Two-Degree-of-Freedom Servo Motor Speed Compensation}

The concept of 2DOF feedback control has been widely applied for the drive system design. The commonly used control schemes are shown in the dashed line circle of Figure 2(b). Two feedback loops and two blocks are added to achieve the performance. With the two inserted blocks, $G_{c 1}$ 


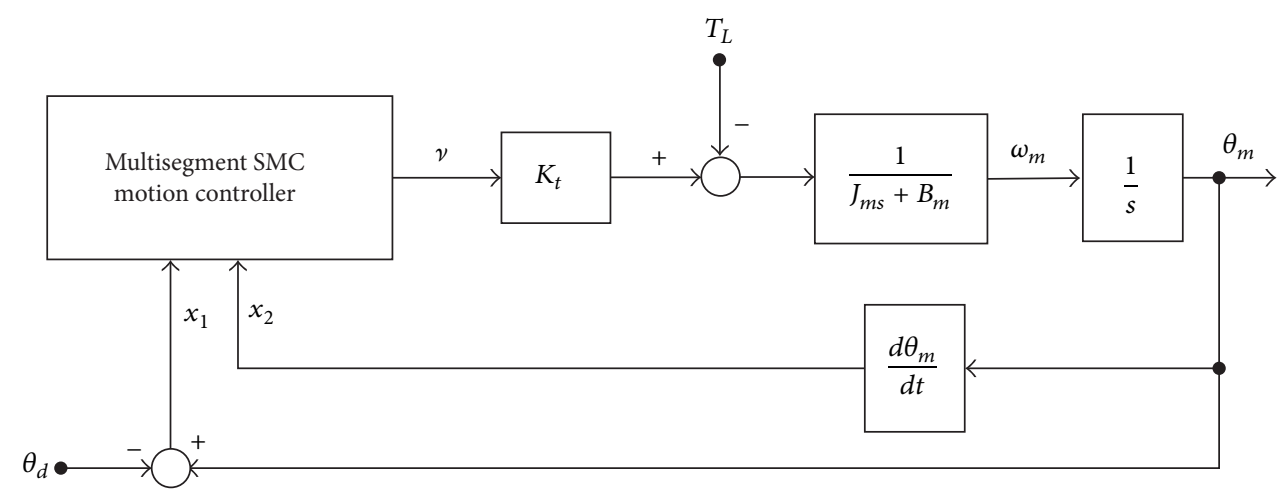

(a)

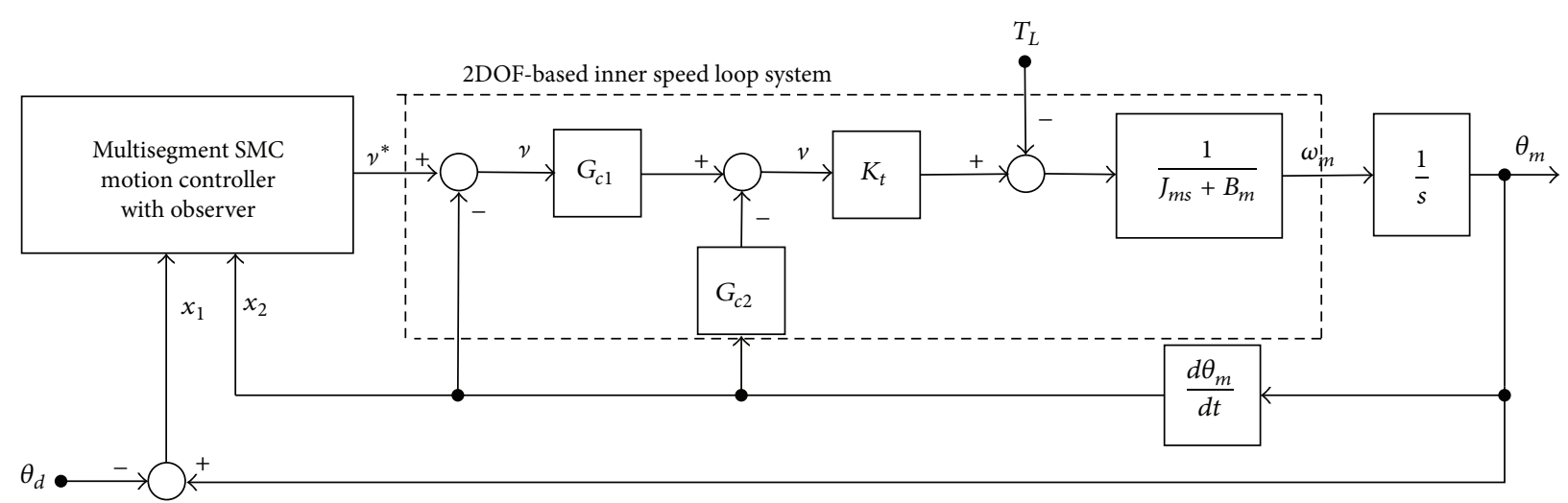

(b)

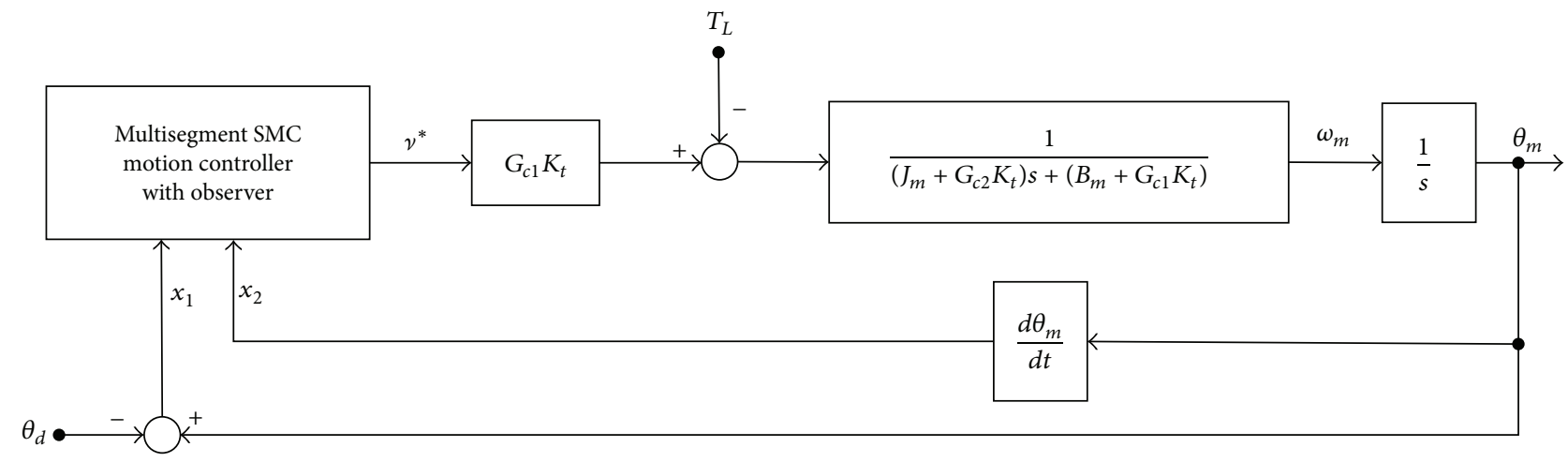

(c)

FIGURE 2: The block diagrams of multisegment motion control scheme: (a) the system without the 2DOF speed loop, (b) the system with 2DOF speed loop, and (c) the simplified block diagram of scheme (b).

and $G_{c 2}$, the system transfer function of speed control loop is first altered to be

$$
H_{1}(s) \triangleq \frac{\Omega_{m}(s)}{V^{*}(s)}=\frac{G_{c 1}(s) G_{p}(s)}{1+G_{c 1}(s) G_{p}(s)+G_{c 2}(s) G_{p}(s)},
$$

where $V^{*}(s)$ is the SMC controller output while $G_{p}(s)$ is the controlled motor. The inserted two blocks and feedback loops are designed to compensate the system uncertainties and external disturbances. To reach this goal, these two control blocks are chosen, respectively, as

$$
\begin{gathered}
G_{c 1}(s)=k_{1}, \\
G_{c 2}(s)=s k_{2},
\end{gathered}
$$

where $k_{1}$ and $k_{2}$ are two constant gains. To substitute (13) and controlled motor transfer function

$$
G_{p}(s)=\frac{\Omega_{m}(s)}{V(s)}=\frac{K_{t}}{J_{m} s+B_{m}}
$$


into (12), we have

$$
\begin{aligned}
H_{1}(s)= & k_{1} \frac{K_{t} / J_{m}}{s+B_{m} / J_{m}} \\
& \times\left(1+k_{1} \frac{K_{t} / J_{m}}{s+B_{m} / J_{m}}+s k_{2} \frac{K_{t} / J_{m}}{s+B_{m} / J_{m}}\right)^{-1} \\
= & \frac{k_{1}\left(K_{t} / J_{m}\right)}{\left(s+B_{m} / J_{m}\right)+k_{1}\left(K_{t} / J_{m}\right)+s k_{2}\left(K_{t} / J_{m}\right)} .
\end{aligned}
$$

As shown in Figure 2(b), one places the 2DOF scheme into the motion system as the inner speed loop and makes the multisegment sliding mode motion controller as the outer loop. In the following, we just consider the compensated results of the inner loop. Motor systems, in (1), with uncertainties and without the external load are rewritten as

$$
\left(\bar{J}_{m}+\Delta J_{m}\right) \frac{d \omega_{m}}{d t}+\left(\bar{B}_{m}+\Delta B_{m}\right) \omega_{m}=T_{e}
$$

and the inner speed control loop transfer function, in (15), can be transformed as

$$
H_{1}(s)=\frac{k_{1} K_{t}}{s\left[\left(\bar{J}_{m}+\Delta J_{m}\right)+k_{2} K_{t}\right]+\left[\left(\bar{B}_{m}+\Delta B_{m}\right)+k_{1} K_{t}\right]} .
$$

If the two parameters, $k_{1}$ and $k_{2}$, are chosen large enough, and with a rather quantity of torque constant, $K_{t}$, the following two inequalities will be true:

$$
\begin{aligned}
& k_{1} K_{t} \gg\left(\bar{B}_{m}+\Delta B_{m}\right), \\
& k_{2} K_{t} \gg\left(\bar{J}_{m}+\Delta J_{m}\right) .
\end{aligned}
$$

Then the system characteristics, in (17), are less relative to the uncertainties. It can also be approximated as follows:

$$
\begin{aligned}
& H_{1}(s) \\
& \quad=\frac{k_{1} K_{t} /\left(\left(\bar{J}_{m}+\Delta J_{m}\right)+k_{2} K_{t}\right)}{s+\left(\left(\bar{B}_{m}+\Delta B_{m}\right)+k_{1} K_{t}\right) /\left(\left(\bar{J}_{m}+\Delta J_{m}\right)+k_{2} K_{t}\right)} \\
& \cong \frac{\left(k_{1} / k_{2}\right)}{s+\left(k_{1} / k_{2}\right)} .
\end{aligned}
$$

Equation (19) has a dc gain of $H_{1}(0)=1$, and it demonstrates the benefit of system dominated by $2 \mathrm{DOF}$ system that the resulting system is nearly invariant or less relative to the system's nominal parameters, $K_{t}, \bar{J}_{m}$, and $\bar{B}_{m}$, and the corresponding uncertainties, $\Delta J_{m}$ and $\Delta B_{m}$.

To consider the effects from external disturbance, $T_{L}$, we have the following transfer function for the inner speed loop as Figure 2(c) shows:

$$
\begin{aligned}
\frac{\Omega_{m}(s)}{T_{L}(s)} & =\frac{1}{\left(J_{m}+K_{t} k_{2}\right) s+\left(B_{m}+K_{t} k_{1}\right)} \\
& =\frac{1 /\left(J_{m}+K_{t} k_{2}\right)}{s+\left(B_{m}+K_{t} k_{1}\right) /\left(J_{m}+K_{t} k_{2}\right)} .
\end{aligned}
$$

Equation (20) illustrates a dc gain of $1 /\left(B_{m}+K_{t} k_{1}\right)$. Comparing it to the system without $2 \mathrm{DOF}$ structure, its $\mathrm{dc}$ gain is $1 / B_{m}$. The magnitude of external disturbance is reduced by inner 2DOF loop. SMC-based motion control system with the 2DOF scheme added thus needs a less magnitude chatter control to make the sliding mode exist. Because the added 2DOF system can improve the robustness of the sliding mode control system, it compensates the drawbacks due to the usage of observer.

\section{The Simulation Results}

To demonstrate the effectiveness of the proposed control strategy, simulations are done by SIMNON software. The velocity profile for simulation is, respectively, set as follows [3]: $\alpha_{d 1}=165 \mathrm{rad} / \mathrm{sec}^{2}, \omega_{d}=30 \mathrm{rad} / \mathrm{sec}$, and $\alpha_{d 3}=$ $165 \mathrm{rad} / \mathrm{sec}^{2}$, and the position control mode is achieved by making $c_{4}=5$. The motor's parameters are severally $\bar{J}_{m}=$ $0.002 \mathrm{Nm} / \mathrm{sec}^{2}, \bar{B}_{m}=0.0015 \mathrm{Nm} / \mathrm{sec}$, and $K_{t}=2.25 \mathrm{Nm} / \mathrm{v}$. Furthermore, two kinds of perturbations are assumed: (1) an external load of $2 \mathrm{Nm}$ is added at $0.2 \mathrm{sec}$ and removed at $1.2 \mathrm{sec}$; (2) the motor parameter with uncertainties, $J_{m}=$ $10 \bar{J}_{m}$ and $B_{m}=2 \bar{B}_{m}$. For those conditions being set, the control gains for each segment can be decided according to the maximum values: $J_{m \max }=0.02 \mathrm{Nm} / \mathrm{sec}^{2}, B_{m \max }=$ $0.003 \mathrm{Nm} / \mathrm{sec}$, and $T_{L \max }=2.0 \mathrm{Nm}$ [3]. In addition, we use the speed observer, $H_{\text {so }}(z)$, in which

$$
H_{\text {so }}(z)=\frac{1+z^{-1}+z^{-2}+z^{-3}+z^{-4}}{5},
$$

to compute the motor's rotating velocity from the encoder pulse. To realize the block $G_{c 2}$, a high-pass filter

$$
G_{c 2}(s)=\frac{100 s}{s+100}
$$

is used, and its discrete equivalent, $G_{c 2}(z)$, as shown in (23), is programmed as follows:

$$
G_{c 2}(z)=\frac{100 z-100}{z-0.9802}
$$

Likewise, to obtain the equivalent control law, a perturbation estimator,

$$
\frac{d}{d t} v^{*}=-50 v^{*}+50 v
$$

is used. All of the equivalent digital filters are simulated on sampling frequency of $5 \mathrm{kHz}$. Figures 3 and 4 are the simulated results. Figure 3 is under nominal condition, whereas Figure 4 is with load and parameter perturbations. In Figures 3 and 4, plots (a) and (b) are, respectively, the position and velocity responses, while (c) and (d) are the motor driver input and the output of multisegment SMC controller. Figures 3(d) and 4(d) show that high frequency chattering exists in the control laws and the equivalent controls, $v^{*}$, are estimated by the perturbation estimator, in (24), as shown in Figures 3(e) and 4(e). It is apparent that the control law is completely 


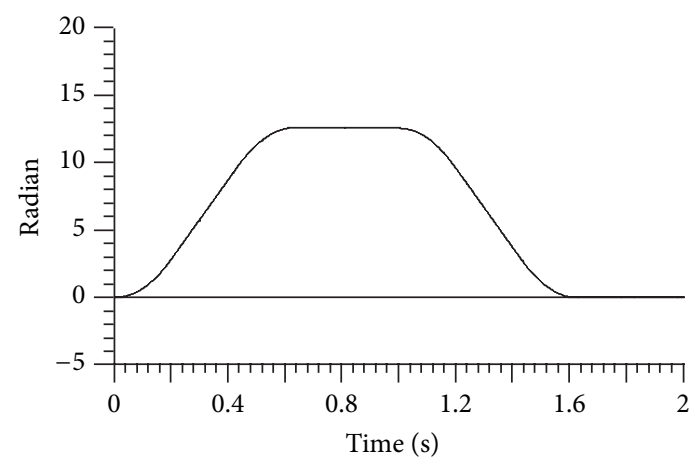

(a)

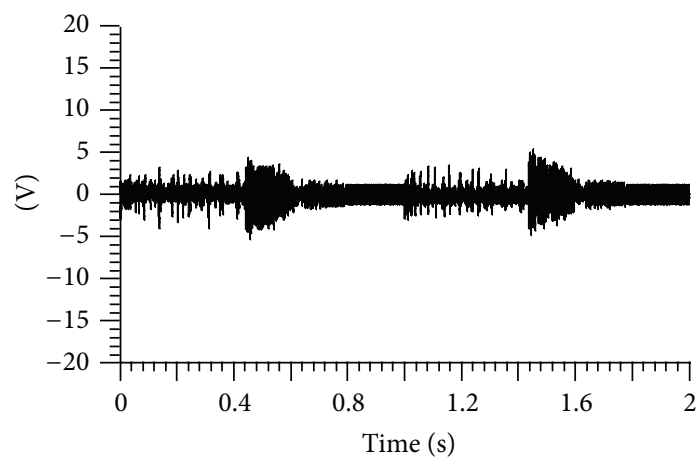

(c)

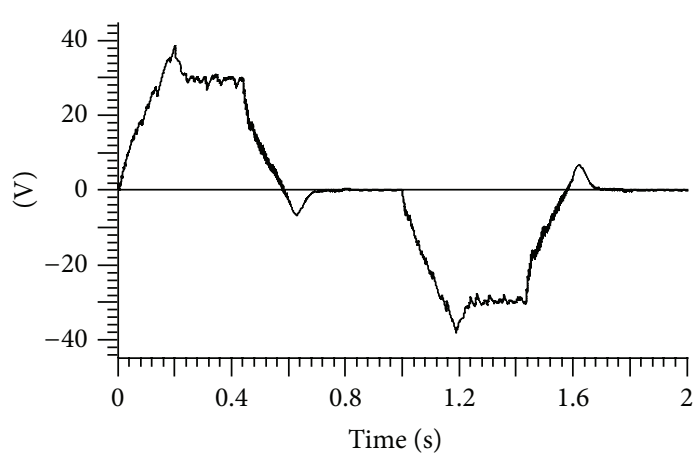

(e)

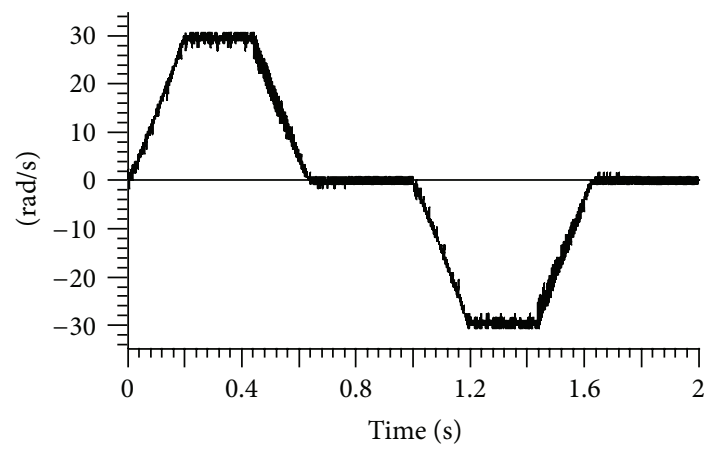

(b)

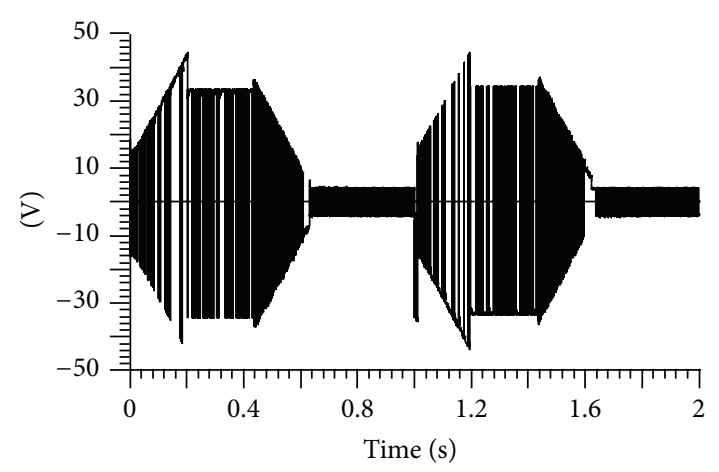

(d)

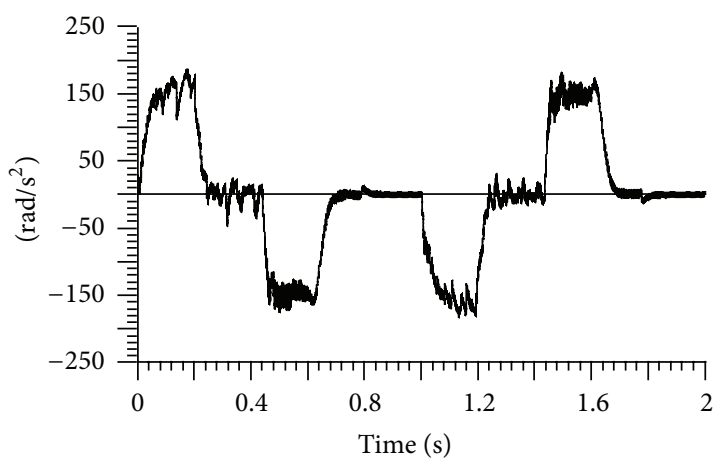

(f)

FIGURE 3: The simulated results for nominal system. (a) The position response, (b) the speed response, (c) the motor driver input, (d) the multisegment SMC controller output, (e) the perturbation observer output, and (f) the estimation of acceleration.

a smooth function and the chattering is reduced. Figures 3(f) and $4(f)$ are, respectively, the estimated motor acceleration. It shows that the added 2DOF control block and perturbation estimator truly lower the system chattering and maintain the robustness.

\section{The Experimental Setup and Results}

6.1. Experimental System Setup. To practically evaluate the performance of the proposed control scheme, a prototype PMSM motion control system is built and examined. The realized system is composed of a PC, an A/D-D/A card with a 16-bit up/down counter, and a $750 \mathrm{~W}$ PMSM. The multisegment control algorithms, perturbation observer, and
2DOF controller are implemented by the PC. The position signals are sensed by a 2000 pulse/rev encoder and feedback to PC through a 16-bit up/down counter with four-time precision multiplied. The corresponding mechanical velocity is computed in PC through the difference in encoder pulse per sampling with fifth-order smooth average as (21) shows. The acceleration estimator is accomplished by two cascaded first-order discrete-type high-pass filters with the transfer function as follows:

$$
H_{\mathrm{hp}}(z)=\frac{100 z-100}{z-0.9802}
$$

where the estimator input comes from the encoder pulse. The recognized system for experimentation is shown in Figure 5. 


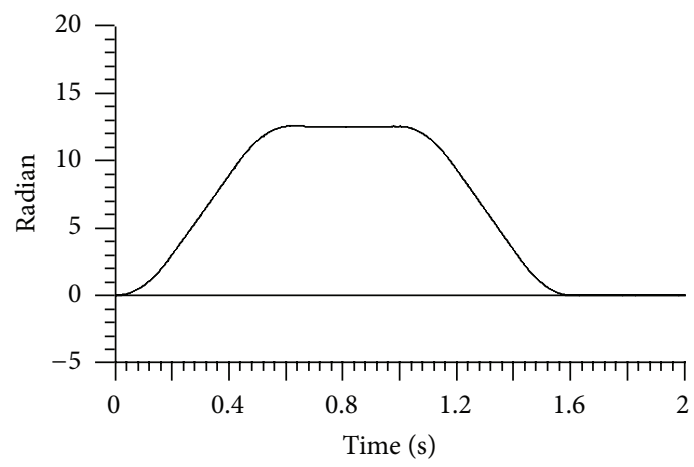

(a)

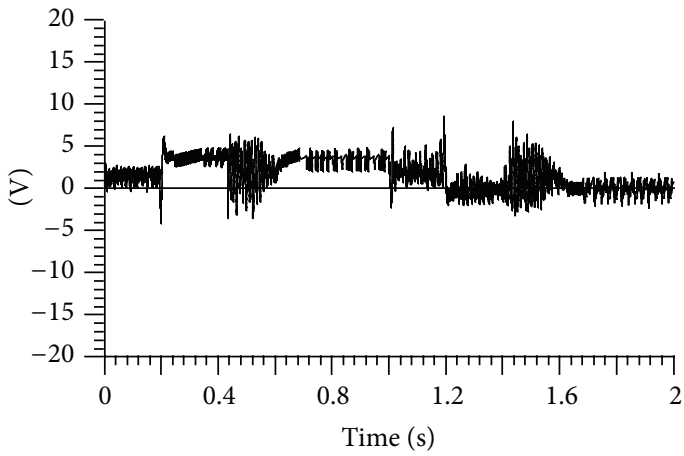

(c)

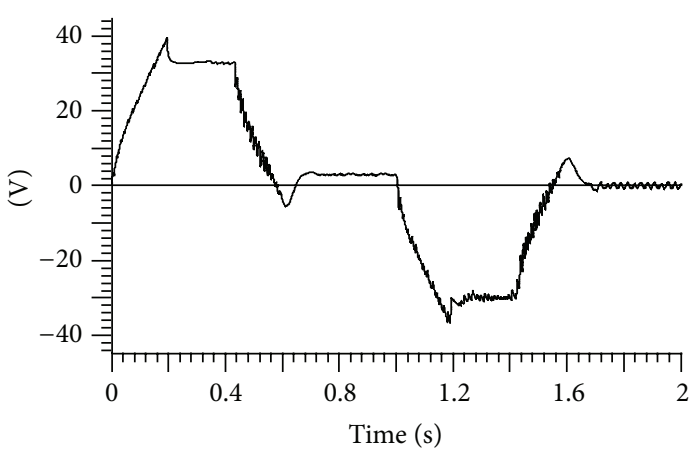

(e)

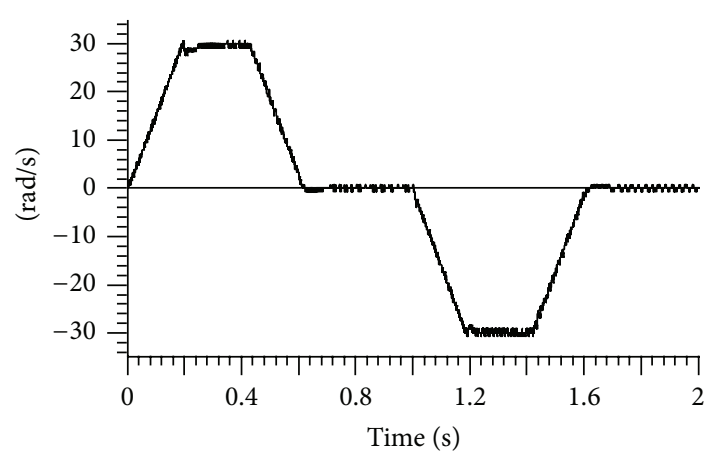

(b)

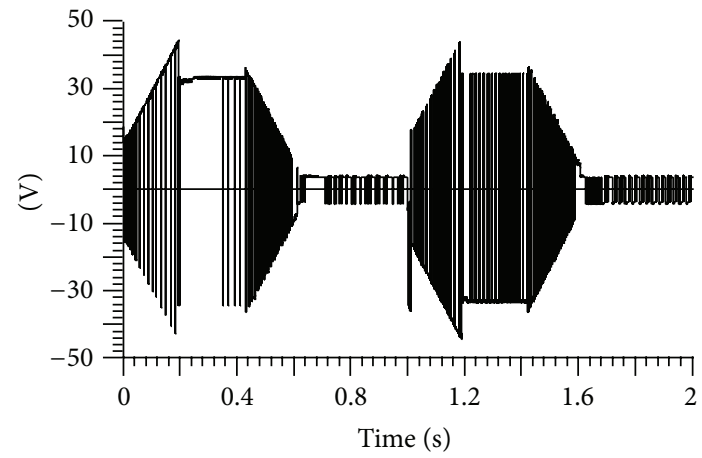

(d)

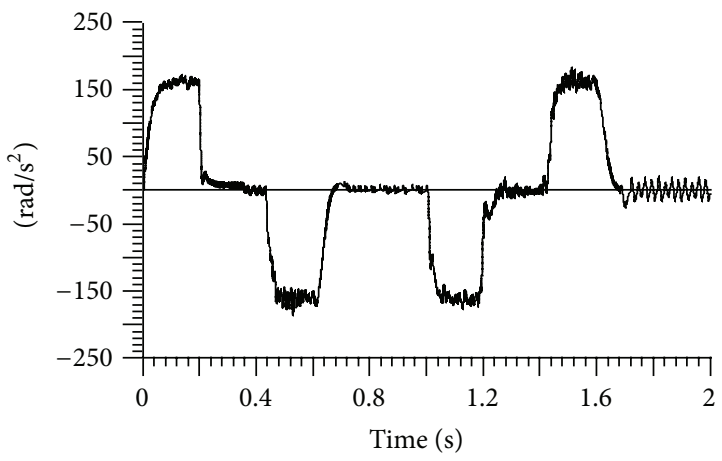

(f)

FIGURE 4: The simulated results for system with load and parameter uncertainties. (a) The position response, (b) the speed response, (c) the motor driver input, (d) the multisegment SMC controller output, (e) the perturbation observer output, and (f) the estimation of acceleration.

The main program for managing data input and output is programmed by assembly language, and control system including all the calculation is implemented on PC. A hardware timer which supports $5 \mathrm{kHz}$ interrupt request is served as the sampling rate and is used to periodically trigger the PC-based controller and run the control algorithms. The data of experimental results, including the acceleration $\alpha_{d}$, the velocity $\omega_{m}$, the position $\theta_{m}$, the control laws $\nu$, and the estimated equivalent control $\nu^{*}$, are directly collected in PC,and processed and printed out through MATLAB. To show the impactions from external load, the PMSM is axially coupled to another motor. And the resulting system has the moment of inertia of 10-time the nominal condition, and the damping coefficient of twice the nominal condition.
6.2. Experimental Results. The actual experimental results are shown in Figures 6 and 7 where the control parameters are set as those in the simulation. Each of the experimental processes is running twice and the results are accumulated in the second time. Figure 6 demonstrates the experimental results of motion control without the motor load, while Figure 7 shows the results under the coupled motor load.

Figures $6(\mathrm{a})$ and $7(\mathrm{a})$ are the responses of position with the position command $4 \pi$ radian forward running at time $0 \mathrm{sec}$ and then backward running to the original position at time $2 \mathrm{sec}$. Figures 6(b) and 7(b) are their corresponding velocity. It is obvious that the velocity responses for both with and without motor load show good results as the programmed and the simulated results revealed on the last 


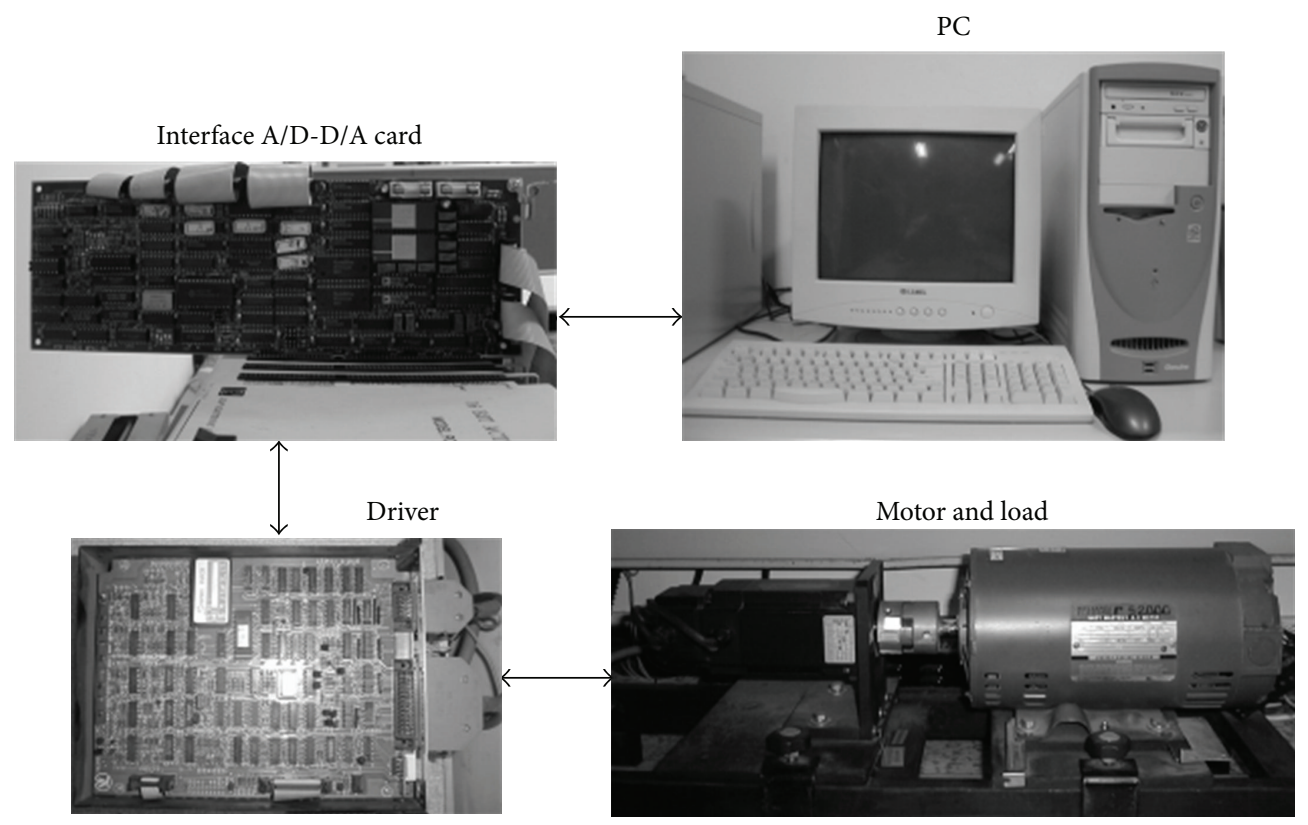

FIGURE 5: The experimental setup.

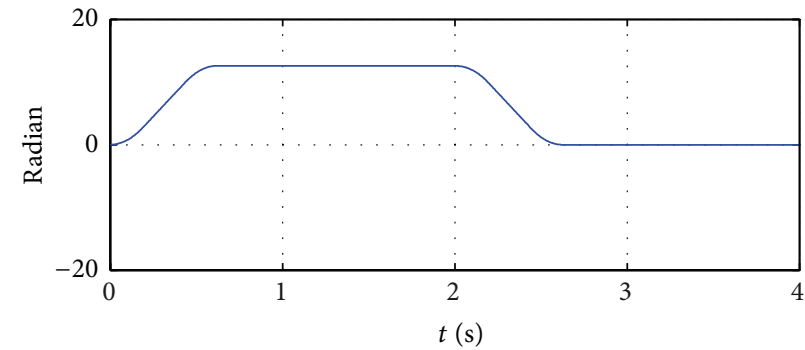

(a)

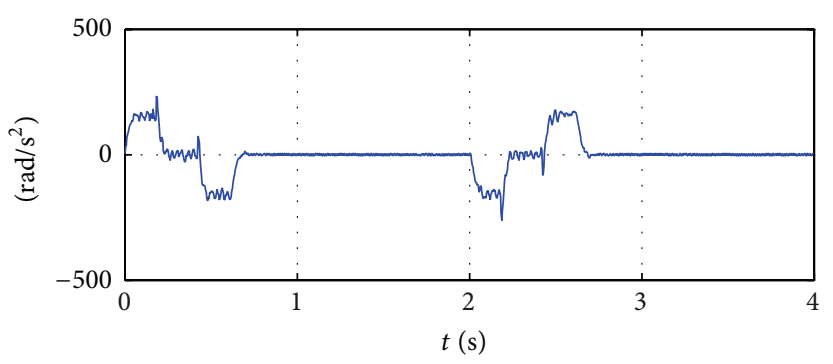

(c)

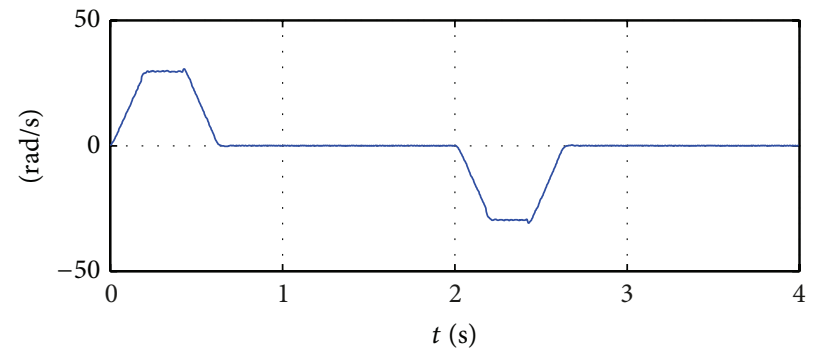

(b)

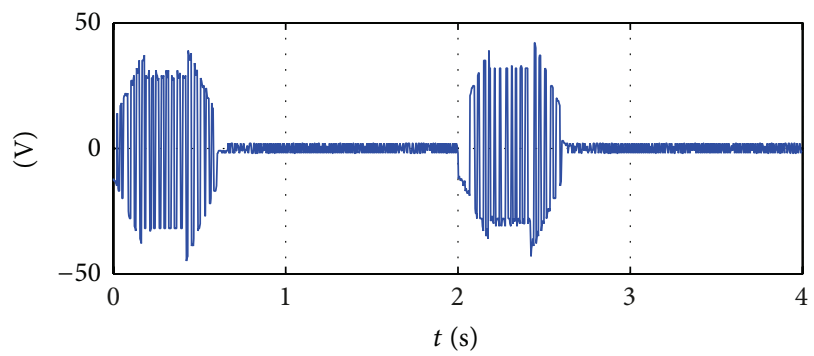

(d)

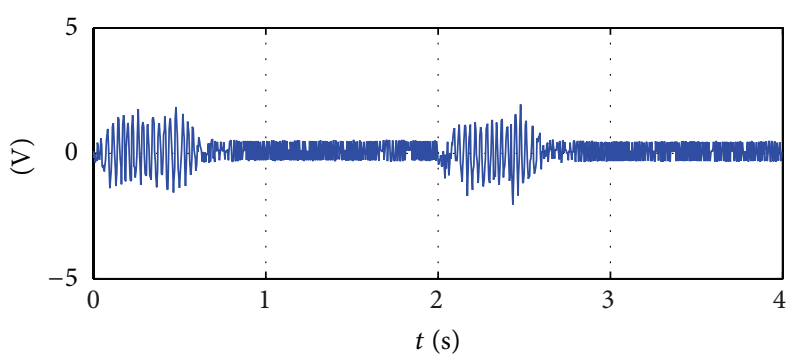

(e)

FIGURE 6: The experimental results of nominal condition. (a) The position responses, (b) the velocity responses, (c) the estimated acceleration, (d) the multisegment sliding mode controller output, and (e) the 2DOF controller output. 


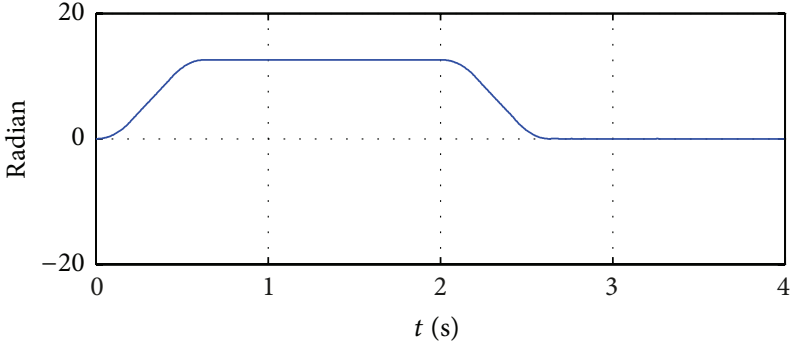

(a)

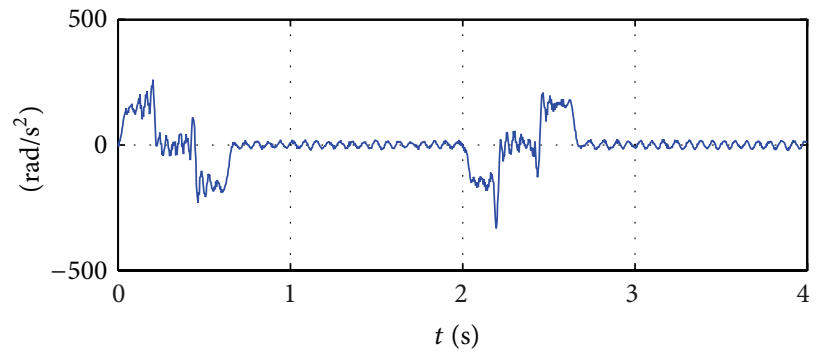

(c)

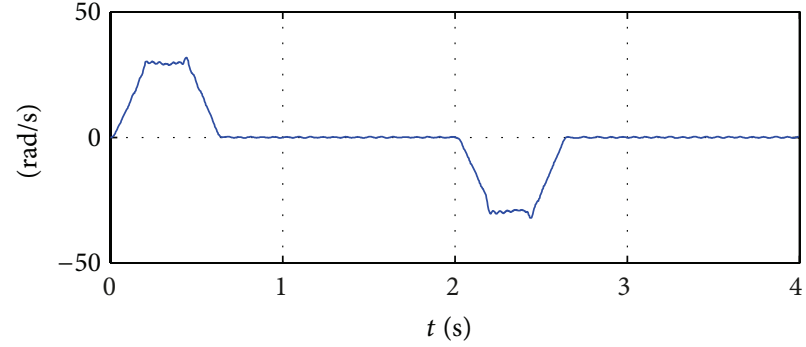

(b)

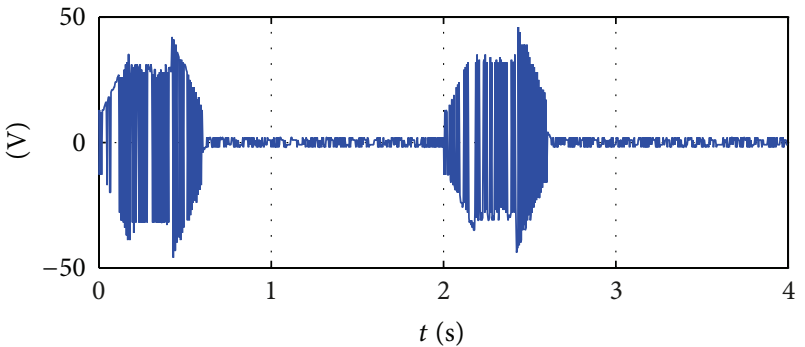

(d)

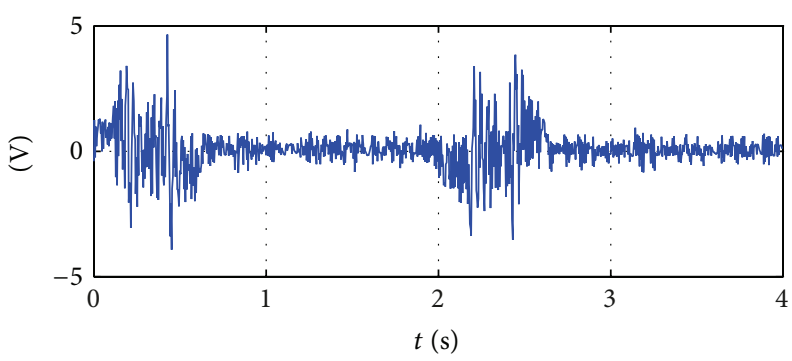

(e)

FIGURE 7: The experimental results of system with external load. (a) The position responses, (b) the velocity responses, (c) the estimated acceleration, (d) the multisegment sliding mode controller output, and (e) the 2DOF controller output.

section. Figures 6(c) and 7(c) are the estimated acceleration during the process under the cascaded second-order highpass filter of (25). As the setting in the simulation, the programmed acceleration and deceleration rates are $165 \mathrm{rad} / \mathrm{sec}^{2}$ and $-165 \mathrm{rad} / \mathrm{sec}^{2}$, respectively, and the experimental acceleration results of Figures 6(c) and 7(c) indicate that the desired acceleration profiles are achieved despite the fact that the little ripples exist at the steady-state condition.

Figures 6(d) and 7(d) illustrate the control laws from the output of multisegment sliding mode controller. The style of control law of multisegment sliding mode control is designated by (6), (8), and (10). Comparing the simulated and experimental results in Figures 3(d), 4(d), 6(d), and 7(d), they all display that the chattering controls exist at the output of sliding mode control. To reduce the impact of chattering phenomenon, a perturbation estimator shown in (24) is designed to estimate the equivalent control law, minimize the amplitude of control law, and maintain the motor rotor following the desired track. Owing to the low-pass characters of perturbation estimator, the motor motion control under the trapezoid velocity profile has to alter its acceleration rate whenever the control segments change over. The $2 \mathrm{DOF}$ controller is thus applied to compensate the weakness and improve the robustness of motion controller. Figures 6(e) and 7 (e) are the control laws made by the $2 \mathrm{DOF}$ control loop. The control command of $2 \mathrm{DOF}$ control loop is from the output of perturbation estimator. Because the designed 2DOF control scheme can compensate the system uncertainty and external disturbance, the needed control law from the SMC controller is thus reduced. The problem of chattering and robustness caused by the high gain control of SMC and perturbation observer is therefore overcome by the added 2DOF controller. The control results shown in Figures 6(a) and 7(a) for both systems with and without perturbation fit the expectation.

\section{Conclusions}

In this paper, the multisegment SMC and 2DOF controllers are used to complete the motion control under the constraint of trapezoid velocity profile. The motor rotor velocity and acceleration control are programmed by the multisegment SMC controller, and the chattering phenomenon from the high gain setting is reduced by a perturbation observer. And the inner 2DOF control scheme is used to make up 
the robustness due to the adoption of perturbation observer. The simulation and experimental results confirm the validness.

\section{Conflict of Interests}

The author declares that there is no conflict of interests regarding the publication of this paper.

\section{References}

[1] J. Y. Hung, W. Gao, and J. C. Hung, "Variable structure control. A survey," IEEE Transactions on Industrial Electronics, vol. 40, no. 1, pp. 2-22, 1993.

[2] W.-D. Chou, F.-J. Lin, and K.-K. Shyu, "Incremental motion control of an induction motor servo drive via a geneticalgorithm-based sliding mode controller," IEE Proceedings: Control Theory and Applications, vol. 150, no. 3, pp. 209-220, 2003.

[3] K.-K. Shyu and C.-K. Lai, "Incremental motion control of synchronous reluctance motor via multisegment sliding mode control method," IEEE Transactions on Control Systems Technology, vol. 10, no. 2, pp. 169-176, 2002.

[4] T. H. Liu and M. T. Lin, "A fuzzy sliding-mode controller design for a synchronous reluctance motor drive," IEEE Transactions on Aerospace and Electronic Systems, vol. 32, no. 3, pp. 10651076, 1996.

[5] R. J. Wai and F. J. Lin, "Fuzzy neural network sliding-mode position controller for induction servo motor drive," IEE Proceedings Electric Power Applications, vol. 146, no. 3, pp. 297-308, 1999.

[6] K. Jezernik, J. Korelic, and R. Horvat, "PMSM sliding mode FPGA-based control for torque ripple reduction," IEEE Transactions on Power Electronics, vol. 28, no. 7, pp. 3549-3556, 2013.

[7] X. Zhang, L. Sun, K. Zhao, and L. Sun, "Nonlinear speed control for PMSM system using sliding-mode control and disturbance compensation techniques," IEEE Transactions on Power Electronics, vol. 28, no. 3, pp. 1358-1365, 2013.

[8] C. Zhang, L. Jia, and J. He, "Load torque observer based sliding mode control method for permanent magnet synchronous motor," in Proceedings of the 25th Chinese Control and Decision Conference (CCDC '13), pp. 550-555, May 2013.

[9] J. Y. Hung and J. C. Hung, "Chatter reduction in variable structure control," in Proceedings of the 20th International Conference on Industrial Electronics, Control and Instrumentation, pp. 19141918, September 1994.

[10] N. Khaled and A. R. Ofoli, "An Incremental Sliding Mode Controller (ISMC) for chattering reduction," in Proceedings of the 46th IEEE Industry Applications Society Annual Meeting (IAS '11), pp. 1-5, Orlando, Fla, USA, October 2011.

[11] K. D. Young, V. I. Utkin, and Ü. Özgüner, "A control engineer's guide to sliding mode control," IEEE Transactions on Control Systems Technology, vol. 7, no. 3, pp. 328-342, 1999.

[12] W. Wang, "New gain-scheduling sliding mode controller for permanent magnet synchronous motor system," in Proceedings of the Asia-Pacific Power and Energy Engineering Conference (APPEEC '11), pp. 1-4, Wuhan, China, March 2011.

[13] H.-S. Lim, S.-C. Shin, H.-J. Lee, T.-K. Lee, and C.-Y. Won, "A comparison of position controllers for IPMSM using sliding mode controller," in Proceedings of the International Conference on Electrical Machines and Systems (ICEMS '11), pp. 1-6, Beijing, China, August 2011.

[14] D. L. Zhang, Y. P. Chen, J. M. Xie, W. Ai, and C. M. YUan, "A hybrid control method of sliding mode and PID controllers based on adaptive controlled switching portion," in Proceedings of the 29th Chinese Control Conference (CCC '10), pp. 439-445, Beijing, China, July 2010.

[15] P. Huang, L. Huang, and C. Miao, "Study on multi-segment sliding-mode-control-based servo system," in Proceedings of the International Conference on Electrical Machines and Systems (ICEMS '10), pp. 844-847, Incheon, Republic of Korea, October 2010. 


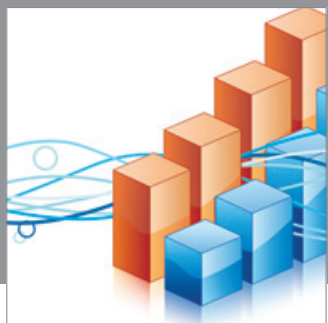

Advances in

Operations Research

mansans

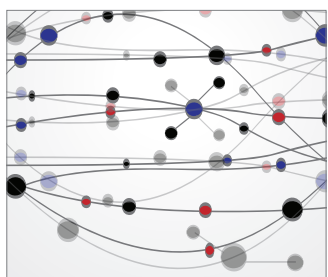

The Scientific World Journal
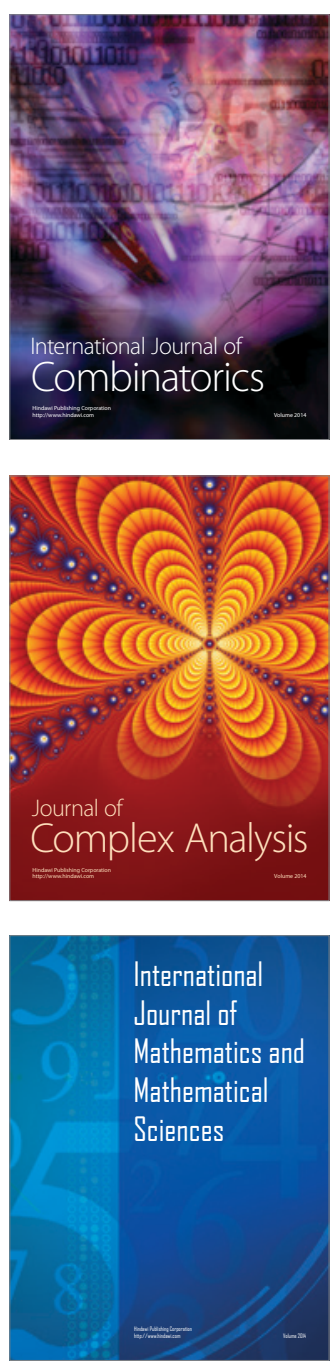
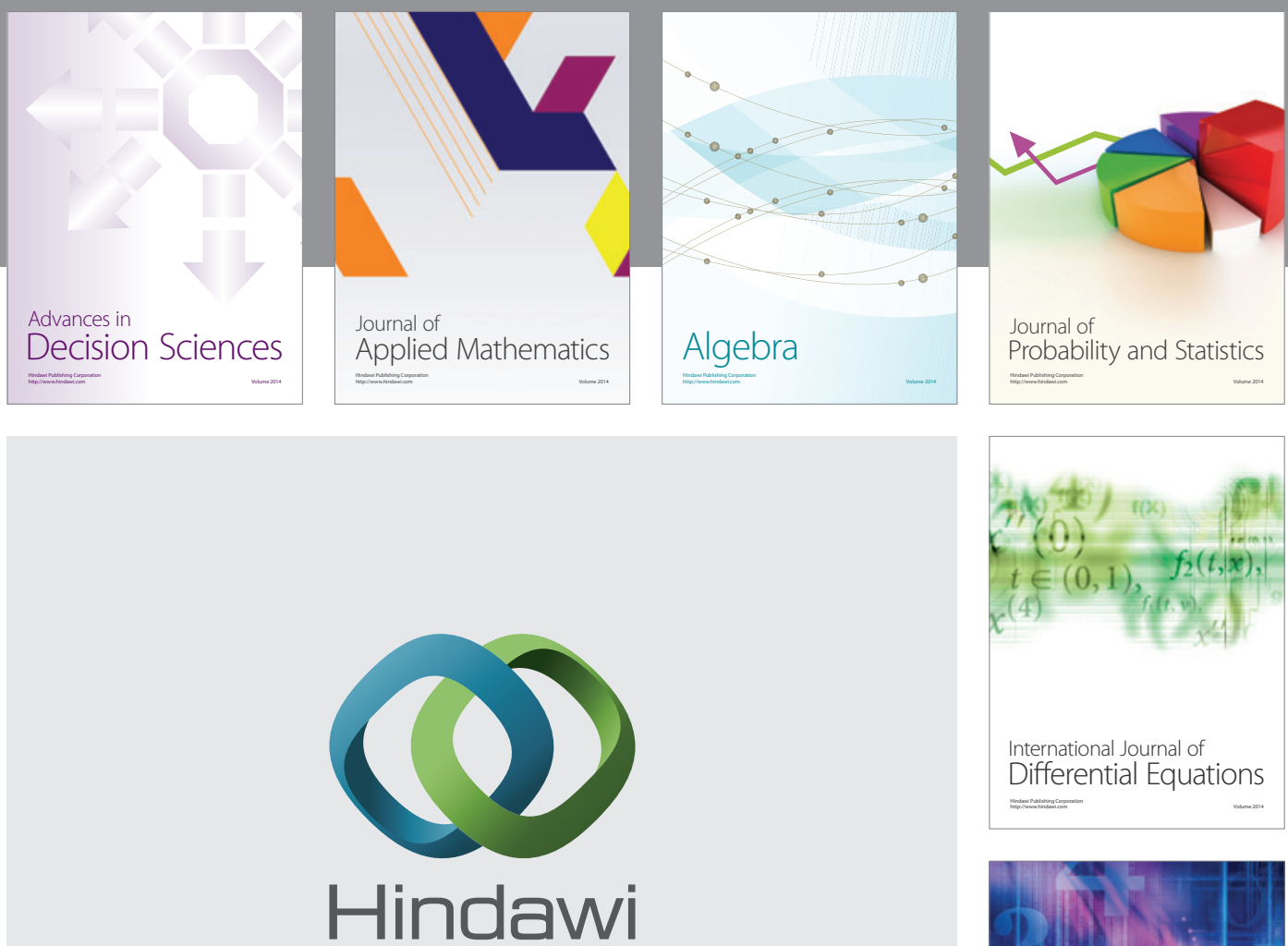

Submit your manuscripts at http://www.hindawi.com
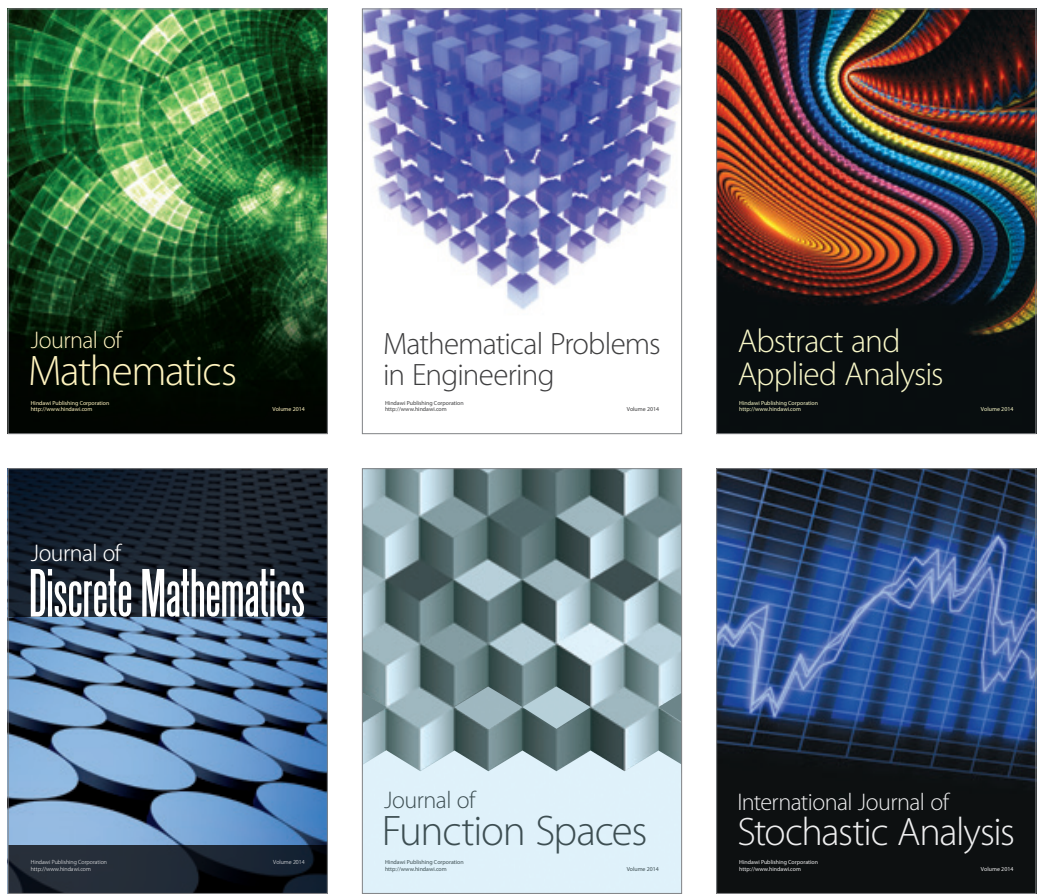

Journal of

Function Spaces

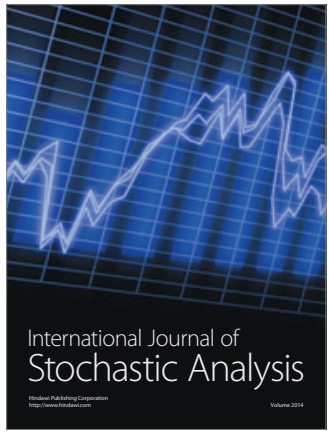

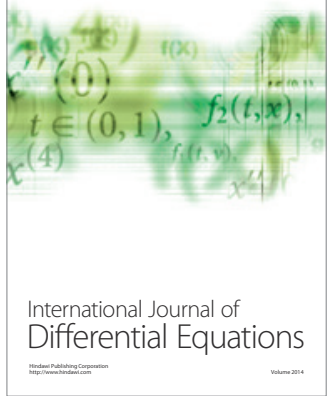
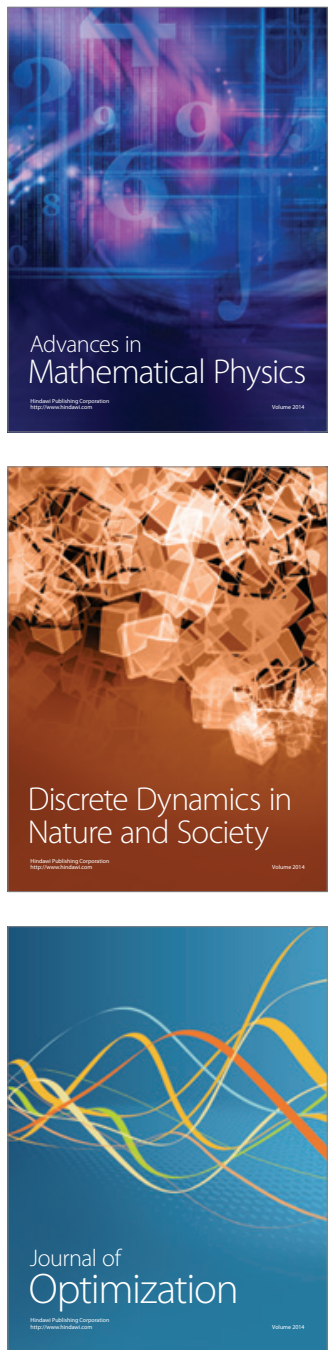